Brock Education

Vol 14, No. 1, 2004

\title{
L'évaluation du concept de soi chez les enfants du préscolaire
}

\author{
Yves Herry \\ Claire Maltais \\ Université d'Ottawa \\ Normand Gingras \\ Université d'Ottawa
}

\begin{abstract}
Résumé
Le présent article s'intéresse à l'évaluation du concept de soi des jeunes enfants fréquentant les classes du préscolaire. Il propose une analyse psychométrique de trois instruments de mesure du concept de soi, soit le Puppet Interview (Verschueren, Marcoen, et Schoefs, 1996), le Pictorial Self-Evaluation Scale (Verschueren et Marcoen, 1993; Verschueren et al., 1996) et une version adaptée par Marsh, Craven, et Debus (1991) du Self-Description Questionnaire-I. Pour chaque instrument, il présente une analyse factorielle, ainsi que le calcul de la cohérence interne et de l'indice de fiabilité testretest. De plus, puisque le Puppet Interview permet une classification des enfants au sein de quatre catégories de concepts de soi, il inclut une comparaison des distributions obtenues par cette étude à celle obtenue par Verschueren et al. (1996). Pour le Puppet Interview, les résultats indiquent que la cohérence interne, la fiabilité test-retest et la cohérence de la classification sont faibles. De plus, l'analyse factorielle ne confirme pas la structure proposée par Verschueren et al. (1996). Cependant, le Pictorial Self-Evaluation Scale et le Self-Description Questionnaire-I démontrent une fiabilité et une cohérence interne satisfaisantes. La structure factorielle du Pictorial Self-Evaluation Scale demeure unifactorielle comme l'avaient prévu Verschueren et al. (1996). Cependant, l'échelle du concept de soi global du Self-Description Questionnaire-I inclurait deux facteurs au lieu d'être unidimensionnelle comme le prévoyaient Marsh et al. (1991).
\end{abstract}

Le monde de l'éducation reconnaît l'importance du concept de soi et de sa relation avec le développement global de l'enfant (Harter, 1990). Les programmes scolaires insistent sur cette relation et prévoient des objectifs pédagogiques visant ce champ de développement. Les programmes destinés aux enfants du préscolaire n'échappent pas à cette tendance. En effet, les dernières refontes des programmes scolaires destinés aux études préscolaires (ministère de l'Éducation

Yves Herry et Claire Mattais, Faculté d'éducation, Université d'Ottawa. Normand Gingras, Départment de psychologies, Université d'Ottawa. 
du Québec, 2000; ministère de l'Éducation de l'Ontario, 1998) insistent sur l'importance du concept de soi dans le développement personnel et social du jeune enfant. Toutefois, peu d'études ont porté sur le concept de soi des enfants du préscolaire. Les recherches sur le concept de soi ont souvent exclu cette clientèle à cause de la difficulté posée par l'évaluation de leur concept de soi (Harter, 1986). En effet, même si au cours des années 80, les chercheurs ont mis au point plusieurs instruments valides pour étudier le concept de soi (Harter, 1982; Marsh, 1985), la plupart s'adressait aux enfants de plus de huit ans. Chez les jeunes enfants, la « complexité » de la composante verbale de ces tests rendait difficile la compréhension des énoncés et de la tâche demandée.

Cependant, au cours des années 90, des chercheurs (Eder et Mangelsdorf, 1997; Harter, 1996; L'Écuyer, 1994; Marsh et al, 1991; Verschueren et al., 1996) ont créé ou adapté des instruments visant l'évaluation du concept de soi des jeunes enfants.

Le présent article s'intéresse à l'évaluation du concept de soi global des jeunes enfants fréquentant les classes du préscolaire. Il propose une analyse psychométrique de trois instruments de mesure de nature quantitative visant l'évaluation du concept de soi global des jeunes enfants. Ces trois instruments sont le Puppet Interview (Verschueren et al., 1996), le Pictorial Self-Evaluation Scale (Verschueren et Marcoen, 1993; Verschueren et al., 1996) et une version adaptée par Marsh et al. (1991) du Self-Description Questionnaire-I. Le choix de ces instruments repose sur leur fréquence d'utilisation dans les recherches sur le concept de soi, sur leur possibilité d'utilisation auprès de jeunes populations et finalement sur la possibilité de comparer la fidélité et la validité de ces instruments. Les trois instruments retenus ont déjà fait l'objet d'une analyse psychométrique par leurs auteurs, sans que leurs résultats aient toutefois été corroborés par d'autres études. De plus, ces tests font appel à des tâches de nature variée. Le Puppet Interview est une entrevue semi-structurée pendant laquelle l'examinateur pose des questions sur l'enfant à une marionnette qui est manipulée par l'enfant. Le Pictorial Self-Evaluation Scale demande à l'enfant de choisir entre deux énoncés, chacun représenté par une illustration, celui qui le représente le mieux. Finalement, la version adaptée par Marsh et al. (1991) du Self-Description Questionnaire-I demande à l'enfant de répondre oralement en indiquant par « oui » ou par «non » si les énoncés le décrivent bien.

L'article s'intéresse particulièrement au Puppet Interview, car la tâche à accomplir paraît originale et confère à l'enfant un rôle actif lors de l'entrevue. De plus, la forme de l'instrument (une entrevue à l'aide d'une marionnette) s'adresse exclusivement aux jeunes enfants, contrairement à celle des deux autres instruments qui sont dérivées d'instruments de mesure destinés aux enfants plus âgés. Finalement, la fidélité et la validité des versions du Pictorial Self- 
Evaluation Scale et du Self-Description Questionnaire-I destinés aux enfants âgés entre 8 et 12 ans ont fait l'objet de plusieurs analyses qui les ont jugés satisfaisantes, même si les qualités psychométriques des versions destinées aux jeunes enfants sont moins connues. Les prochaines sections décrivent les trois instruments.

\section{Le Puppet Interview}

Le Puppet Interview (Verschueren et al., 1996) est une entrevue individuelle. Elle comprend 20 questions au sujet de l'enfant qui sont posées à une marionnette que manipule l'enfant. L'enfant répond donc aux questions par l'intermédiaire de la marionnette. Selon Verschueren et al. (1996, p. 2495), «The answers the child gives through the hand puppet are assumed to reveal the child's perceptions of how an unspecified other views him or her, and hence reveal the child's own level of self-esteem. ». Les auteures considèrent que cette stratégie d'évaluation rend l'enfant plus actif tout en faisant appel à son quotidien. Les réponses des enfants sont enregistrées et transcrites de façon verbatim.

Le Puppet Interview a été développé par Cassidy (1988). La version originale permettait de classer les concepts de soi des enfants en deux catégories, soit « high-quality », regroupant les enfants qui avaient un concept de soi positif et ouvert aux imperfections, et « low-quality », regroupant les enfants dont le concept de soi était négatif ou parfait (non ouvert aux imperfections). Dans leur version, Verschueren et al. (1996; Verschueren, Schoefs, et Marcoen, 1994) ont voulu tenir compte, dans un premier temps, de ces deux dimensions séparément (la polarité et l'ouverture à l'imperfection) et, dans un deuxième temps, de la combinaison de ces deux dimensions pour obtenir quatre types de concepts de soi: (a) positif-ouvert, (b) positif-parfait, (c) négatif-ouvert, (d) négatif-parfait.

Des 20 questions de l'entrevue, 15 visent l'évaluation de la polarité du concept de soi. Voici des exemples de questions posées aux enfants: «Bic (nom de la marionnette), est-ce que tu aimes <nom de l'enfant>? » «Aimes-tu <nom de l'enfant> comme il est ou tu aimerais qu'il soit meilleur? » « Dis-moi Bic, voudrais-tu que <nom de l'enfant> soit ton ami? ». Les réponses aux questions sont analysées à l'aide d'une grille fournie par Verschueren et al. (1994) qui permet de déterminer si l'énoncé est positif, semi-négatif ou négatif. Les critères couvrent toutes les réponses possibles fournies par l'enfant. La codification individuelle des réponses aux 15 questions permet de déterminer si le concept de soi est positif ou négatif. Le concept de soi est considéré négatif si l'enfant 
formule un énoncé négatif à une de ces 15 questions ou s'il formule deux énoncés semi-négatifs; sinon il est considéré positif.

Les cinq autres énoncés de l'entrevue visent l'évaluation de l'ouverture aux imperfections. Voici des exemples de questions posées aux enfants: «Y a-t-il quelque chose qui pourrait être meilleur chez $<$ nom de l'enfant>? » Si oui, «quoi? » «Est-ce que c'est déjà arrivé que tu ne sois pas content de <nom de l'enfant>? » Si oui: «Quand est-ce que c'est arrivé? Qu'est-ce qui s'est passé? ». Les réponses aux questions sont analysées à l'aide d'une grille fournie par Verschueren et al. (1994) qui permet de déterminer si la réponse est ouverte aux imperfections ou si la réponse indique que l'enfant se considère parfait. Les critères couvrent toutes les réponses possibles fournies par l'enfant. Le concept de soi est considéré ouvert si l'enfant reconnaît au moins une imperfection à une des cinq questions; sinon il est considéré parfait. Les règles d'interprétation des énoncés liés à l'ouverture sont beaucoup plus élaborées et complexes que celles visant la polarité. Elles sont toutefois claires et précises.

Verschueren et al. (1996) ne fournissent pas d'information sur la structure factorielle des deux échelles. Ils indiquent toutefois que la corrélation entre les deux échelles est faible $(0,15, \mathrm{~ns})$, soulignant l'indépendance des deux échelles. De plus, les auteurs présentent des informations concernant la fidélité de l'interprétation des réponses fournies par les enfants. La fidélité inter-juges est de 0,87 et la corrélation entre les deux cotes (two raters' scores) est de 0,92 pour la polarité et 0,96 pour l'ouverture à l'imperfection.

\section{Le Pictorial Self-Evaluation Scale}

Le Pictorial Self-Evaluation Scale (Verschueren et Marcoen, 1993; Verschueren et al., 1996) est un instrument d'évaluation du concept de soi dérivé du Pictorial Self of Perceived Competence and Social Acceptance for Young Children (Harter et Pike, 1984), lui-même dérivé du Self-Perception Profile for Children (SPPC) (Harter, 1982, 1985). Ce dernier test a été grandement utilisé au cours des quinze dernières années lors des recherches sur le concept de soi des enfants âgés entre 8 et 12 ans. Dans un premier temps, Harter et Pike (1984) ont adapté le SPPC pour les jeunes enfants en diminuant le nombre d'énoncés de 36 à 24 et le nombre de domaines évalués de six à quatre. Cependant, le recours à des illustrations au lieu d'énoncés écrits constitue la plus importante modification qu'elles ont apportée à cet instrument. Les analyses factorielles effectuées par Harter et Pike (1984), n'ont pas pu démontrer l'existence des quatre facteurs ou domaines que prévoyait couvrir l'instrument de mesure. Ces résultats ont conduit Verschueren et son équipe (Verschueren et Marcoen, 1993; 
Verschueren et al., 1996) à produire une version simplifiée du test de Harter et Pike (1984) en n'incluant que six énoncés visant l'évaluation du concept de soi global des jeunes enfants.

Le nouvel instrument conserve la même méthodologie que celui de Harter et Pike (1984). Chaque énoncé est représenté par deux illustrations, une illustrant son pôle positif (ex. cet enfant joue bien avec ses amis) et l'autre son pôle négatif (ex. cet enfant se dispute avec ses amis). Pour chacun des énoncés, l'enfant doit choisir l'illustration qui le caractérise le plus et préciser si l'enfant représenté sur l'illustration lui ressemble beaucoup ou un peu. Chaque énoncé correspond à une échelle à quatre points allant de un à quatre; un étant le pôle négatif et quatre le pôle positif. L'enfant obtient un résultat moyen variant de un à quatre qui correspond à la moyenne des résultats obtenus aux six énoncés.

Les analyses psychométriques réalisées par Verschueren et al. (1996) rapportent des coefficients alpha de cohérence interne Cronbach variant entre 0,82 (première administration) et 0,71 (deuxième administration). De plus, une analyse factorielle de type LISREL révèle que les six énoncés peuvent être considérés comme un seul facteur $\left(\mathrm{X}^{2}(9)=6,83, \mathrm{p}=0,65\right.$, GFI $\left.=0,98\right)$ avec des coefficients de saturation variant entre 0,57 et 0,81 . La corrélation test-retest est 0,38 .

\section{Le Self-Description Questionnaire-I}

Le Self-Description Questionnaire-I (1988) permet l'évaluation du concept de soi des enfants âgés entre huit et douze ans. Il constitue l'instrument de mesure quantitative du concept de soi le plus utilisé à l'heure actuelle. Il inclut 102 énoncés permettant l'évaluation de sept domaines du concept de soi, en plus du concept de soi global. En 1991, Marsh et al. (1991) ont proposé de modifier les procédures d'administration de l'instrument afin de permettre son utilisation auprès des enfants âgés entre cinq et huit ans. En s'inspirant du Pictorial Self of Perceived Competence and Social Acceptance for Young Children (Harter et Pike, 1984), ils ont remplacé l'échelle de réponse aux énoncés à cinq points (de faux à vrai) par une échelle à quatre points: non toujours, non parfois, oui parfois, oui toujours. Pour chacun des énoncés lu à voix haute, l'enfant doit indiquer s'il est d'accord ou non avec l'énoncé. Après avoir répondu par oui ou par non, l'enfant doit quantifier sa réponse par toujours ou parfois, en pointant respectivement un grand cercle ou un petit cercle. Pour la présente étude, nous n'avons conservé que l'échelle visant le concept de soi global du Self-

Description Questionnaire-I, puisque les deux autres instruments utilisés (le Puppet Interview et le Pictorial Self-Evaluation Scale) ne visent que le concept de soi global des enfants. Les énoncés sont les suivants: Je fais 
beaucoup de choses importantes; J'ai beaucoup de raisons d'être fier (content) de moi; Je peux faire les choses aussi bien que la plupart des autres; Les autres pensent que je suis une bonne personne; Il y a beaucoup de bonnes choses en moi; Je suis aussi bon(ne) que la plupart des autres; Lorsque je fais quelque chose, je le fais bien; En règle générale, $\mathrm{j}$ ' aime être comme je suis. Les analyses psychométriques réalisées par Marsh et al. (1991) rapportent un coefficient alpha de cohérence interne Cronbach de 0,72.

Les prochaines sections présentent la méthodologie de la recherche, ainsi que les résultats des analyses psychométriques menées sur les trois instruments de mesure retenus. Elles permettront de les comparer et d'identifier les forces et les faiblesses de chaque instrument. Les résultats serviront de guide pour les recherches ultérieures sur le concept de soi des jeunes enfants.

\section{La méthodologie}

\section{Les enfants participant à l'étude}

Deux cent soixante enfants de la maternelle (Québec) et du jardin d'enfants (Ontario) provenant de 5 écoles de l'Outaouais (15 classes) ont participé à cette étude. Ce groupe comprenait 130 filles et 130 garçons. L'âge moyen des enfants était de 72,3 mois. Cent dix-huit enfants ont complété les trois questionnaires. Les autres enfants ont complété deux des trois questionnaires soit le Puppet Interview et le Pictorial Self-Evaluation Scale.

\section{La collecte des données}

La collecte des données prévoyait une rencontre individuelle d'environ 40 minutes par enfant. L'entrevue débutait par le Puppet Interview, suivi du Pictorial Self-Evaluation Scale et du Self-Description Questionnaire-I. Cinq étudiantes au baccalauréat en éducation étaient responsables des entrevues. Elles ont reçu une formation de deux jours visant l'administration des instruments, la transcription des résultats et l'interprétation des réponses des enfants aux questions du Puppet Interview. Ce dernier est l'instrument qui a requis le plus de temps de formation à cause de la complexité de l'interprétation des réponses des enfants.

Trois semaines après la première administration, nous avons procédé à une deuxième administration des trois instruments. Cette deuxième administration visait 45 enfants pour le Puppet Interview, 56 enfants pour le Pictorial Self-Evaluation Scale, et 33 enfants pour le Self-Description Questionnaire-I. 
Des stratégies de contrôle ont été mises en place pour assurer la qualité des informations recueillies. Ces stratégies visaient principalement le Puppet Interview, l'instrument le plus difficile à administrer et à interpréter. La première mesure a consisté à retranscrire dix pour cent des entrevues afin de les comparer avec la première transcription de l'entrevue. La seconde était une vérification systématique par deux évaluateurs externes (fidélité inter-juges) de l'interprétation des réponses des enfants aux questions du Puppet Interview (dix pour cent des enfants). Finalement, nous avons procédé à une vérification de l'entrée des données des trois tests chez dix pour cent des enfants.

\section{Le plan d'analyse des données}

Pour chaque instrument, nous avons procédé à une analyse factorielle, au calcul de la cohérence interne et de l'indice de fiabilité test-retest. De plus, le Puppet Interview permettant la classification des enfants en quatre catégories de concepts de soi, nous avons procédé à une comparaison des distributions obtenues par cette étude à celle obtenue par Verschueren et al. (1996).

\section{Les résultats}

Cette section présente les résultats des analyses effectuées afin de déterminer les qualités psychométriques de chacun des trois instruments de mesure du concept de soi.

\section{Les résultats concernant le Puppet Interview}

Les réponses des enfants aux 20 énoncés du Puppet Interview ont fait l'objet d'analyses variées. Elles incluent des analyses factorielles, des calculs de la cohérence interne et de la corrélation test-retest, de même qu'une comparaison entre les distributions des sujets de cette étude au sein des quatre types de concepts de soi et celles obtenues par Verschueren et al. (1996).

Les analyses factorielles avaient pour but de vérifier la structure des deux échelles théoriquement contenues dans le Puppet Interview, soit la polarité du concept de soi (énoncés 1-5, 8-11,14, 16-20), et l'ouverture aux imperfections (énoncés 6, 7, 12, 13 et 15). Ces deux échelles se sont avérées indépendantes: la corrélation entre elles est faible. Elle se situe à -0,12 (ns) (première administration, $\mathrm{n}=257$ ) et à 0,01 (ns) (deuxième administration, $\mathrm{n}=45$ ). Ces corrélations sont semblables à celles obtenues par Cassidy $(-0,12, n s)(1988)$ et Verschueren et al. $(0,15, \mathrm{~ns})(1996)$. 
Des analyses préliminaires ont évalué la normalité de la distribution des réponses aux énoncés. L'examen des moyennes, des écarts-types, des symétries et des kurtoses révèle que les réponses aux énoncés présentent une dispersion qui dévie fortement de la normale, surtout pour les 15 énoncés visant l'évaluation de la polarité du concept de soi. Selon Gorsuch (1983), des variables dichotomiques ou trichotomiques présentant des kurtoses supérieures à 10 risquent fort de générer des facteurs additionnels factices (« spurious factors »). Or, les valeurs de kurtoses et de symétries des 15 énoncés visant l'évaluation de la polarité varient respectivement entre 0,05 et 16,03 et entre 0,0 et 257,00 . À cause de ces résultats, il sera difficile d'établir la validité de cette échelle. Cependant, le nombre de sujets $(\mathrm{n}=257)$ est jugé suffisant pour mener une analyse factorielle (Tabachnik et Fidell, 1983), surtout à cause de l'homogènéité du groupe et du petit nombre de variables. La dispersion, l'écarttype, les symétries et les kurtoses des cinq énoncés visant l'évaluation de l'ouverture à l'imperfection indiquent un écart acceptable de la normalité, compte tenu de la nature dichotomique des données (Gorsuch, 1983). Pour ces cinq énoncés, les valeurs de kurtoses et de symétries se situent entre -0,47 et 2,00 et entre - 1,00 et 2,00 respectivement. Finalement, l'énoncé 5 ( «Bix, qu'elle est la meilleure chose que tu peux me dire au sujet de <nom de l'enfant $>$ ? ») n'a pu être conservé lors de l'analyse factorielle, car il affichait une variance de zéro. Suite aux analyses préliminaires, les donnés ont été soumises à une analyse factorielle exploratoire de type « Maximum Likelihood » avec rotation orthogonale Varimax. Elle a révélé la présence de sept facteurs expliquant presque les deux tiers $(59,9 \%)$ de la variance et pour lesquels les valeurs propres (« eigen values ») sont supérieures à 1 . De ces sept facteurs, quatre $(12,6 \%$, $10,7 \%, 8,7 \%$, et $8,1 \%)$ expliquent la plus grande partie $(40,2 \%)$ de cette variance. Les coefficients de saturation sont satisfaisants, étant supérieurs à $0,30 . \mathrm{La}$ proportion de variance expliquée par les facteurs (« communalities ») au sein de chaque énoncé est plus petite que un, ce qui indique une solution «nonproblématique » (Tabachnik et Fidell, 1983).

En forçant la pondération à se limiter à deux facteurs (rotation Varimax), la polarité représente 11,1 pour cent de la variance et l'ouverture aux imperfections en représente 12,7 pour cent. La faible variabilité associée aux énoncés caractérisant l'échelle de la polarité, ainsi que les kurtoses et les symétries très élevées, pourraient bien justifier la faible proportion de variance expliquée par les deux facteurs.

Le tableau 1 présente la structure factorielle du Puppet Interview (limitée à deux facteurs). Les énoncés 1, 2, 10, 11 et 14 sont principalement corrélés avec la polarité du concept de soi, tandis que les énoncés 6, 7, 12, 13 et 15 le sont avec l'ouverture aux imperfections. Ces énoncés se comportent 
comme Verschueren et al. (1996) l'avaient prédit. Toutefois, les énoncés 16 et 18, qui devaient être corrélés avec la polarité du concept de soi, sont plutôt fortement corrélés avec l'ouverture aux imperfections. De plus, les énoncés 3, 4, $8,9,17,19$ et 20 (supposés appartenir à l'échelle de la polarité) ne s'associent à aucun des deux facteurs.

\section{Tableau 1}

Analyse factorielle exploratoire du Puppet Interview

\begin{tabular}{|c|c|c|c|}
\hline Énoncés* & $\begin{array}{c}\text { Polarité } \\
(\mathrm{a}=0,31)\end{array}$ & $\begin{array}{l}\text { Ouverture } \\
(\mathrm{a}=0,56)\end{array}$ & $\begin{array}{c}\text { Total } \\
(\mathrm{a}=0,35)\end{array}$ \\
\hline$\overline{1}$ & $-0,70$ & & \\
\hline 2 & $-0,70$ & & \\
\hline 6 & & $-0,62$ & \\
\hline 7 & & $-0,70$ & \\
\hline 10 & $-0,31$ & & \\
\hline 11 & $-0,37$ & & \\
\hline 12 & & $-0,64$ & \\
\hline 13 & & $-0,64$ & \\
\hline 14 & $-0,67$ & & \\
\hline 15 & & $-0,42$ & \\
\hline 16 & & 0,64 & \\
\hline 18 & & 0,43 & \\
\hline variance & $11,06 \%$ & $12,75 \%$ & $23,81 \%$ \\
\hline
\end{tabular}

L'évaluation de la cohérence interne a permis d'établir les coefficients alpha de Cronbach. Le calcul a utilisé tous les énoncés prescrits par Verschueren et al. (1996) pour chaque échelle. Les coefficients alpha se situent respectivement à 0,35 (l'ensemble des énoncés), 0,31 (la polarité), et 0,56

(l'ouverture aux imperfections). Ces valeurs sont peu élevées, sauf celle visant l'ouverture aux imperfections qui est modérée. Les valeurs de cohérence interne obtenues par le groupe d'enfants qui a fait l'objet d'une deuxième administration $(n=45)$ (test-retest) sont semblables à celles obtenues par 
l'ensemble des sujets ( $\mathrm{n}=257)(0,55$ : l'ensemble des énoncés; 0,14 : la polarité; 0,56 : l'ouverture aux imperfections).

Une autre série d'analyses s'intéressait aux répartitions des enfants au sein des quatre types de concepts de soi (positif-ouvert, positif-parfait, négatifouvert, négatif-parfait), tant celle obtenue lors de la première administration $(\mathrm{n}=257)($ respectivement $46 \%, 33 \%, 16 \%$ et $6 \%)$ que celle obtenue lors de la deuxième administration $(\mathrm{n}=45)$ (respectivement $35 \%, 44 \%, 17 \%$ et $4 \%)$. Les tests Chi-carré soulignent que chacune de ces distributions diffère significativement d'une distribution au hasard (respectivement $X^{2}=97,94$, $\left.\mathrm{p}<Q, 001 ; \mathrm{X}^{2}=13,93, \mathrm{p}<0,005\right)$. Cependant, il existe une différence significative $\left(X^{2}=51,48, p<0,001\right)$ entre la distribution des enfants de la première administration et celle obtenue lors de la deuxième administration. De plus, la comparaison entre la distribution des enfants de la présente étude $(n=257)$ avec celle de l'étude de Verschueren et al. (1996) (n=95) (respectivement 34\%, 27\%, $18 \%$ et $21 \%$ ) indique une différence significative entre les deux distributions $\left(X^{2}=16,64, p<0,001\right)$. Il existe aussi une différence significative $\left(X^{2}=36,80\right.$, $\mathrm{p}<0,001)$ entre la distribution des enfants participant à la deuxième administration $(n=45)$ et celle de Verschueren et al. $(n=95)$. Finalement, la distribution obtenue par Yerschueren ne diffère pas significativement d'une distribution au hasard $\left(X^{2}=5,58, p<0,132\right)$. Ces résultats révèlent une cohérence faible de la classification des élèves au sein des quatre types de concept de soi, d'autant plus que la distribution des enfants de notre étude diffère aussi significativement de celle de l'étude de Verschueren.

Les analyses visant le Puppet Interview ont porté sur la fidélité testretest. Pour la polarité du concept de soi, la corrélation entre la première administration et la deuxième est de 0,28 (ns), alors que pour l'ouverture, elle est de 0,44 ( $\mathrm{p}<0,002)$. La fiabilité test-retest des deux échelles est donc faible. Dans un deuxième temps, puisque le Puppet Interview permet de classer les enfants selon quatre types de concepts de soi, il était possible de vérifier la cohérence de la catégorisation entre les deux administrations. La corrélation est de 0,23 (ns), ce qui confirme la faible fiabilité test-retest du processus de classification proposé par le Puppet Interview.

Finalement, les analyses indiquent une fidélité inter-juges $(n=44)$ de 0,97 pour la polarité et de 0,87 pour l'ouverture à l'imperfection. Il semble donc que les consignes d'interprétation des réponses des enfants soient appliquées de façon uniforme par les évaluateurs.

En somme, nos résultats témoignent d'une fiabilité et d'une validité faibles pour le Puppet Interview, d'autant plus que nos résultats diffèrent significativement de ceux d'une étude menée par d'autres chercheurs. Dans l'ensemble, la faible valeur des indices de fiabilité et de validité semble surtout 
attribuable aux caractéristiques psychométriques des 15 énoncés visant l'évaluation de la polarité du concept de soi, l'échelle visant l'ouverture aux imperfections faisant preuve de caractéristiques psychométriques acceptables; celle-ci fait preuve d'une cohérence interne modérée.

\section{Les résultats concernant le Pictorial Self-Evaluation Scale}

Les réponses des enfants aux six énoncés du Pictorial Self-Evaluation Scale ont fait l'objet d'analyses semblables à celles effectuées sur les données recueillies grâce au Puppet Interview, soit une analyse factorielle et des calculs de la cohérence interne et de la corrélation test-retest.

L'analyse factorielle avait pour but de vérifier le caractère unidimensionnel du Pictorial Self-Evaluation Scale. Les analyses préliminaires ont trouvé des symétries et des kurtoses acceptables en plus d'une moyenne $(3,58)$ et d'un écart-type $(0,41)$ qui s'apparentent à ceux obtenus par Verschueren et al. (1996) (Moy=3,37, ÉT=0,60). L'analyse factorielle exploratoire Maximum Likelihood avec rotation orthogonale Varimax a indiqué que les six énoncés sont saturés sur un seul facteur, expliquant 34,9 pour cent de la variance. Les coefficients de saturation varient de 0,45 à 0,67 , ce qui représente un bon taux de saturation sur ce facteur pour chacun des énoncés. Verschueren et al. (1996) rapportent également un seul facteur, avec des coefficients de saturation variant de 0,57 à 0,81 .

Le coefficient alpha de Cronbach est de 0,62. Chaque énoncé contribue positivement à l'instrument, avec une corrélation moyenne test-énoncé de 0,22. La cohérence interne obtenue lors de la deuxième évaluation (alpha $=0,78)$ est supérieure à celle obtenue lors de la première administration. Verschueren et al. (1996) rapportent un alpha de Cronbach de 0,82 (n=95).

La fiabilité test-retest, calculée en tenant compte du résultat global de chaque sujet, est jugée acceptable avec une corrélation entre les deux administrations de $0,59(\mathrm{p}<0,001)$. Verschueren et al. (1996) rapportent une fiabilité test-retest de 0,38 (0,50 lorsque corrigée pour l'atténuation). La correction pour l'atténuation est une mesure prise pour déterminer la « vraie » fiabilité, lorsqu'on soupçonne qu'un effet de gain a eu lieu entre les deux administrations d'un même test (comme un test visant les habiletés mathématiques) (Bernier, 1985). Nous n'avons pas utilisé cette correction lors de nos calculs, car il est peu probable qu'il y ait un tel gain au niveau de la mesure du concept de soi.

Les six énoncés du PSES présentent donc une fiabilité moyenne et une cohérence interne acceptable. L'analyse factorielle démontre un seul facteur, sur lequel sont saturés tous les énoncés. 


\section{Les résultats concernant le Self-Description Questionnaire-I}

Les réponses des enfants aux huit énoncés de l'échelle du Self-Description Questionnaire-I visant l'évaluation du concept de soi global, ont fait l'objet d'une analyse factorielle et des calculs de la cohérence interne et de la corrélation test-retest.

L'analyse factorielle avait pour but de vérifier la nature unidimensionnelle de l'échelle. Les analyses préliminaires ont trouvé des symétries et des kurtoses acceptables, ainsi que des corrélations inter-énoncé excédant 0,30 , ce qui permet de soumettre les données à une analyse factorielle (Tabachnik et Fidell, 1983). Une analyse factorielle exploratoire Maximum Likelihood avec rotation orthogonale Varimax a indiqué la présence de deux facteurs (F1= énoncés 1, 2, 3 et 6; F2= énoncés 4, 5, 7 et 8) au lieu d'un facteur comme le prévoyaient Marsh et al. (1991). Ces deux facteurs représentent respectivement 27,5 et 23,8 pour cent de la variance (total $=51,3 \%$ de variance expliquée). Les coefficients de saturation varient de 0,60 à 0,83 pour le premier facteur et de $-0,56$ à $-0,73$ pour le deuxième. Ces résultats indiquent un bon taux de saturation pour chacun des énoncés sur ces facteurs (Tableau 2). Alors que cette saturation sur deux facteurs ne semble pas concorder avec l'hypothèse théorique qui veut que les énoncés du questionnaire se réfèrent à un seul concept de soi global, on remarque que dans leur analyse LISREL, Marsh et al. (1991) ont combiné les énoncés 1 et 2, 3 et 4, 5 et 6 , et 7 et 8 . Cette procédure réduit le nombre d'énoncés de huit à quatre et, selon Marsh et al. (1991), aurait pour effet d'augmenter la stabilité de la solution.

\section{Tableau 2}

Analyse factorielle exploratoire du SDQ-I

\begin{tabular}{lccc}
\hline Enoncés & $\begin{array}{c}\text { Facteur I } \\
(a-0,66)\end{array}$ & $\begin{array}{c}\text { Facteur } 2 \\
(a-0,67)\end{array}$ & $\begin{array}{c}\text { Total } \\
(\mathrm{a}-0,72)\end{array}$ \\
\hline 1 & 0,60 & & \\
2 & 0,62 & & \\
3 & 0,68 & & \\
4 & & $-0,67$ & \\
5 & & $-0,75$ & \\
6 & 0,83 & & \\
7 & & $-0,73$ & \\
8 & & $-0,56$ & \\
\hline variance & $27,50 \%$ & $23,90 \%$ & $51,40 \%$ \\
\hline
\end{tabular}


En reprenant l'analyse factorielle en jumelant les énoncés comme l'ont fait Marsh et al. (1991), on retrouve un seul facteur dont les saturations varient de 0,70 à 0,84 , expliquant 58,1 pour cent de la variance. Si cette technique augmente la puissance et la stabilité de la solution (Marsh et al., 1991), les résultats de nos analyses démontrent qu'elle peut masquer la présence de facteurs sous-jacents. Il faut ajouter que Marsh et al. (1991) ne se sont pas préoccupés de la possibilité de sous-facteurs, car ils ont expressément forcé chacune des huit échelles du SDQ-I à se saturer sur un seul facteur.

Pour évaluer cet effet de masquage, nous avons repris l'analyse factorielle (rotation Varimax) en combinant les énoncés 1 et 2, 3 et 6, 4 et 5, 7 et 8 , selon leur pondération sur l'un ou l'autre des facteurs. Cette analyse révèle deux facteurs expliquant 73,8 pour cent de la variance avec les énoncés 1-2 et 3-6 ayant une saturation très forte sur le premier facteur $(0,81$ et 0,84 respectivement) et les énoncés 4-5 et 7-8 ayant une saturation très forte sur le deuxième $(0,77$ et 0,90 respectivement). La combinaison des énoncés améliore donc sensiblement la proportion de variance expliquée, ce qui confirme l'effet de masquage lié à cette procédure.

Le coefficient alpha de Cronbach pour l'ensemble des énoncés est de 0,74 . Chaque énoncé contribue positivement à l'instrument, avec une corrélation moyenne test-énoncé de 0,27 . Le coefficient obtenu lors de la deuxième administration est légèrement moins élevée $(0,63)$ que lors de la première administration. Marsh et al. (1991) rapportent un coefficient alpha de 0,73 pour l'échelle visant le concept de soi global, résultat semblable à celui obtenu par l'ensemble des enfants de la présente étude. Finalement, la fiabilité test-retest est considérée satisfaisante avec une corrélation entre les deux administrations de $0,72(\mathrm{p}<0,000)$.

L'échelle du SDQI composée de huit énoncés visant l'évaluation du concept de soi global présente une fiabilité et une cohérence interne satisfaisante. Cependant, contrairement aux résultats de Marsh et al. (1991), l'analyse factorielle démontre qu'elle n'est pas unidimensionnelle, mais se compose plutôt de deux facteurs. La technique de combinaison des énoncés utilisée par Marsh et al. (1991) masque la présence de ces deux facteurs. Néanmoins, nos résultats démontrent que les enfants de 4 à 6 ans en maternelle peuvent s'exprimer sur leur concept de soi global.

\section{Discussion des résultats}

Le présent article s'intéressait à l'évaluation du concept de soi des jeunes enfants fréquentant les classes du préscolaire. Il proposait une analyse psychométrique de trois instruments de mesure du concept de soi, soit le 
Puppet Interview (Verschueren et al., 1996), le Pictorial Self-Evaluation Scale (Verschueren et Marcoen, 1993; Verschueren et al., 1996) et une version adaptée par Marsh et al. (1991) du Self-Description Questionnaire-I. Pour chaque instrument, il a présenté une analyse factorielle, ainsi que le calcul de la cohérence interne et de l'indice de fiabilité test-retest. De plus, puisque le Puppet Interview permet une classification des enfants au sein de quatre catégories de concepts de soi, il a inclus une comparaison des distributions obtenues par cette étude à celle obtenue par Verschueren et al. (1996). Le tableau 3 résume les résultats obtenus.

\section{Tableau 3}

\begin{tabular}{|c|c|c|c|c|c|c|c|c|}
\hline & \multicolumn{4}{|c|}{ Puppet interview } & \multicolumn{2}{|c|}{$\begin{array}{c}\text { Pictonial Self. } \\
\text { Evaluation Scale }\end{array}$} & \multicolumn{2}{|c|}{$\begin{array}{l}\text { Seif-Description } \\
\text { Questiannaire-f }\end{array}$} \\
\hline & \multicolumn{2}{|c|}{ Herry et al. } & \multicolumn{2}{|c|}{ Versch. et al. } & \multirow{2}{*}{$\begin{array}{c}\text { Herry } \\
\text { et al. }(2004)\end{array}$} & \multirow{2}{*}{$\begin{array}{c}\text { Versch. } \\
\text { et al. (1996) }\end{array}$} & \multirow{2}{*}{\begin{tabular}{|c|} 
Herry \\
et al. (2004)
\end{tabular}} & \multirow{2}{*}{$\begin{array}{c}\text { Marsh } \\
\text { et al. (1991) }\end{array}$} \\
\hline & Pol. & Ouv. & Pol. & Ouv. & & & & \\
\hline $\begin{array}{l}\text { Cohêrence } \\
\text { interne } \\
\text { (alpha) }\end{array}$ & $\begin{array}{l}, 31 \text { te } \\
, 1420\end{array}$ & $\begin{array}{l}, 5610 \\
, 5620\end{array}$ & $\begin{array}{l}\text { nd } \\
\text { nd }\end{array}$ & $\begin{array}{l}\text { nd } \\
\text { nd }\end{array}$ & $\begin{array}{l}, 6810 \\
, 7820\end{array}$ & 82 & $\begin{array}{l}.7410 \\
.632 e\end{array}$ &, 73 \\
\hline $\begin{array}{l}\text { Corrélations } \\
\text { Test-restest }\end{array}$ & 28 &, 44 & nd & nd &, 59 &, 38 & 72 & ND \\
\hline $\begin{array}{l}\text { Fidélité } \\
\text { inter-juges } \\
\text { (correlations) }\end{array}$ & 97 & .87 & ,92 & 96 & Sio & S/O & Sio & SNo \\
\hline $\begin{array}{l}\text { analyses } \\
\text { factorielles : } \\
\text { nb de facteurs }\end{array}$ & & & nd : : & terés & 1 & 1 & 2 & 1 \\
\hline \multicolumn{9}{|c|}{$\begin{array}{l}\text { Pol. = Polarité } \\
\text { Ouv, = Ouverture aux imperfections } \\
1 \mathrm{e}=\text { première administration } \\
2 \mathrm{e}=\text { deuxième administration } \\
\text { nd }=\text { indéterminée } \\
\mathrm{S} / \mathrm{O}=\text { Sans objet }\end{array}$} \\
\hline
\end{tabular}

Résumé des résultats des analyses statistiques

L'examen des résultats permet de conclure que la fiabilité et la validité du Puppet Interview sont faibles. Cette faiblesse semble surtout attribuable aux caractéristiques psychométriques des 15 énoncés visant l'évaluation de la polarité du concept de soi; l'échelle visant l'ouverture aux imperfections faisant preuve de caractéristiques psychométriques modérées. De plus, l'analyse 
factorielle a mis en évidence sept facteurs au lieu des deux que devait théoriquement contenir l'instrument. Lorsque l'analyse factorielle était limitée à deux facteurs, ceux-ci n'expliquaient qu'à peine plus de 20 pour cent de la variance et plusieurs énoncés n'étaient corrélés avec aucun des deux facteurs ou se corrélaient avec le mauvais facteur. Nous avons même tenté de créer de nouvelles échelles en n'utilisant que les énoncés qui se corrélaient avec les «bons » facteurs, mais les nouvelles corrélations test-retest et les coefficients de cohérence interne ne se sont pas avérés plus satisfaisants que ceux des échelles originales.

Les résultats décevants du Puppet Intewrview pourraient s'expliquer en partie par la variance très faible des valeurs de chaque énoncé. Celle-ci pourrait être attribuable à la formulation des questions et au peu de nuance accordée à l'interprétation des réponses des enfants. En effet, les questions obligent souvent les enfants à répondre par oui ou par non (surtout pour les questions visant la polarité du concept de soi), sans leur laisser la possibilité de nuancer leurs réponses. De plus, nous pensons qu'il faudrait réduire le nombre d'énoncés visant l'évaluation de la polarité, en ciblant mieux le concept de soi global.

Les modifications proposées aux énoncés du Puppet Interview et aux règles d'interprétation des réponses des enfants auront des répercussions sur les règles de classification des enfants au sein des quatre types de concepts de soi développés par Verschueren et al. (1996). La très faible cohérence de cette classification doit conduire à une redéfinition des règles permettant l'identification d'un concept de soi positif et d'un concept de soi ouvert aux imperfections. La révision des énoncés et des règles d'interprétation des réponses des enfants devraient permettre une classification plus cohérente.

Les résultats du Puppet Interview sont décevants car plusieurs éléments jouaient en sa faveur. Les enfants réagissent très bien lors de l'administration de cet instrument basé sur le jeu de rôle. Ils répondent spontanément aux questions et verbalisent bien leurs sentiments. De plus, le Puppet Interview s'est avéré un très bon instrument de mesure du concept de soi des jeunes enfants dans le cadre d'analyses qualitatives des réponses fournies par les enfants. En effet, Herry et Maltais (2001) ont procédé à l'analyse systématique des réponses des enfants à l'aide du modèle expérientiel développé par L'Écuyer (1994) et celles-ci se sont avérées conformes à la structure du concept de soi des enfants de cet âge proposée par l'Écuyer (1994). Ce test s'avère donc prometteur, mais sa réorganisation demanderait une analyse minutieuse de son contenu et de l'interprétation des réponses fournies par les enfants. 
Les résultats concernant les deux autres instruments (le Pictorial SelfEvaluation Scale et le Self-Description Questionnaire-I) sont plus encourageants. Les deux instruments démontrent une fiabilité et une cohérence internes satisfaisantes. Le Pictorial Self-Evaluation Scale démontre une consistance interne moyenne $(0,73)$ légèrement supérieure à celle du SelfDescription Questionnaire-I $(0,68)$, mais celui-ci obtient une meilleure corrélation test-retest (respectivement 0,59 et 0,72 ).

La structure factorielle du Pictorial Self-Evaluation Scale demeure unifactorielle comme l'avaient prévu Verschueren et al. (1996). Cependant, l'analyse factorielle des réponses des enfants aux énoncés de l'échelle du concept de soi global du Self-Description Questionnaire-I a révélé la présence de deux facteurs, au lieu d'un facteur comme le prévoyaient Marsh et al. (1991). Ce résultat est déroutant car l'examen des énoncés qui appartiennent à chaque facteur n'a pas permis d'identifier de thèmes qui regrouperaient les énoncés.

Cet article s'intéressait donc à l'évaluation du concept de soi des enfants. Il indique qu'il est possible d'évaluer le concept de soi global des jeunes enfants et que des instruments comme le Pictorial Self-Evaluation Scale et le Self-Description Questionnaire-I s'avèrent des instruments faciles à administrer, démontrant des qualités psychométriques satisfaisantes. Même si le Puppet Interview ne peut être utilisé dans sa forme actuelle, son concept demeure intéressant, mais son contenu devra faire l'objet d'une refonte en profondeur.

\section{Références}

Bernier, J. J. (1985). Théorie des tests: principes et techniques de base. Chicoutimi: Gaëtan-Morin.

Cassidy, J. (1988). Child-mother attachment and the self in six-year-olds. Child Development, 59, 121-134.

Eder, R. A., \& Mangelsdorf, S. C. (1997). The emotional basis of early personality development: Implication for the emergent self-concept. Dans R. Hogan, \& J. A. Johnson, Handbook of personality psychology (pp. 209-240). San Diego: Academic Press.

Gorsuch, R. L. (1983). Factor analysis. Hillsdale, NJ: Lawrence Erlbaum and Associates.

Harter, S. (1982). The Perceived Competence Scale for Children. Child Development, 53, 87-97.

Harter, S. (1985). Manual for the Self-Perception Profile for Children: Revision of the PCSC (1979). Document non publié. Université de Denver. 
Harter, S. (1986). Processes underlying the construction, maintenance and enhancement of the self-concept. Dans J. Suls, \& A. Greenwald (Eds.), Psychological perspectives on the self (Vol. 3, pp. 136-182). Hillsdale, NJ: Lawrence Erlbaum and Associates.

Harter, S. (1990). Self and identity development. Dans S. Feldman, \& G. R. Elliott (Eds.), At the threshhold: The developing adolescent. Cambridge MA: Harvard University Press.

Harter, S. (1996). Developmental changes in self-understanding across the 5 to 7 shift. Dans A. J. Sameroff, \& M. M. Haith (Eds), The 5 to 7 year shift: The age of reason and responsability (pp. 207-236). Chicago: University of Chicago Press.

Harter, S., \& Pike, R. (1984). The Pictorial Scale of Perceived Competence and Social Acceptance in young children. Child Development, 55, 19691982.

Herry, Y., \& Maltais, C. (2001). Verbalisations à propos du soi chez les enfants de six ans. Document non publié.

L'Écuyer, R. (1994). Le développement du concept de soi de l'enfance à la vieillesse. Montréal: Presses de l'Université de Montréal.

Marsh, H. W. (1985). Self Description Questionnaire-I. Sydney: University of Sydney.

Marsh, H. W. (1988). Self Description Questionnaire: A theorical and empirical basis for the measurement of multiple dimensions of preadolescent self-concept: A test manual and a research mongraph. San Antonio: Psychological Corporation.

Marsh, H. W., Craven, R. G., \& Debus, R. (1991). Self-concepts of young children 5 to 8 years of age: Measurement and multidimensional structure. Journal of Educational Psychology, 83, 377-392.

Ministère de l'Éducation de l'Ontario (1998). Jardin d'enfants. Toronto: Imprimeur de la Reine.

Ministère de l'Éducation du Québec (2000). Programme de formation de l'école québécoise: Éducation préscolaire. Éducation primaire. Québec: ministère de l'Éducation du Québec.

Shavelson, R. J., Hubner, J. J., \& Stanton, G. C. (1976). Self-concept: Validation of construct interpretations. Review of Educational Research, 46, 407441.

Tabachnick, B. G., \& Fidell, L. S. (1983). Using multivariate statistics. New York: Harper and Row.

Verschueren, K., \& Marcoen, A. (1993). The Pictorial Self-Evaluation Scale. Document non-publié. Center for Developmental Psychology, Université de Louvain. 
Verschueren, K., Marcoen, A. \& Schoefs, V. (1996). The internal working model of the self, attachment, and competence in five-year-olds. Child Development, 67, 2493-2511.

Verschueren, K., Schoefs, V., \& Marcoen, A. (1994). Handpop Interview. Instructies en codering. Document non publié. Center for Developmental Psychology, Université de Louvain. 